\title{
Insight and treatment outcome with cognitive-behavioral social skills training for older people with schizophrenia
}

\author{
Lindsay C. Emmerson, PhD; ${ }^{1-2 *}$ Eric Granholm, PhD; ${ }^{1-2}$ Peter C. Link, MA; ${ }^{1}$ John R. McQuaid, PhD; $^{1-2}$ \\ Dilip V. Jeste, MD $^{1-2}$ \\ ${ }^{1}$ Department of Veterans Affairs San Diego Healthcare System, San Diego, CA; ${ }^{2}$ University of California San Diego, \\ San Diego, CA
}

\begin{abstract}
Poor insight (awareness of having a mental illness that requires treatment) is common in schizophrenia and typically predicts poor outcome, yet greater insight has been linked to negative outcomes, including hopelessness. This study focused on two questions: (1) Does insight moderate the effects of cognitive-behavioral social skills training (CBSST) on functional outcomes in schizophrenia? (2) Does a specific type of insight (e.g., awareness of illness, need for treatment) predict benefit from CBSST? We examined insight as a predictor of everyday functioning in a randomized controlled trial of CBSST versus treatment as usual (TAU) for middle-aged and older people with schizophrenia $(n=62)$. We used linear regression models to examine moderators of the relationship between baseline insight and everyday functioning measured 12 months following completion of the 6-month intervention. Insight, especially insight into the need for treatment, moderated the relationship between treatment group and everyday functioning (Independent Living Skills Survey), such that CBSST offset the negative effect of insight on functioning observed with TAU (wherein greater insight was related to poorer everyday functioning). Post hoc analyses showed that reduction of insight-linked hopelessness may have accounted for the positive effect of CBSST on functioning relative to TAU.
\end{abstract}

Clinical Trial Registration: ClinicalTrials.gov; Rehabilitation of Older Patients with Schizophrenia, NCT00018629; http:// www.clinicaltrials.gov/ct $2 /$ show/ NCT00018629?term=Older+Schizophrenia\&rank=7/.
Key words: cognition, functioning, insight, negative symptoms, psychosis, psychosocial, rehabilitation, schizophrenia, social skills, therapy.

\section{INTRODUCTION}

Greater insight is related to improved course of schizophrenia [1], improved social functioning [2-3], decreased rates of rehospitalization, and improved treatment adherence [4-5] but also to greater hopelessness, depressive symptoms [1,6], and suicide risk [7-8]. We previously reported that middle-aged and older people with schizophrenia who received group cognitive-behavioral social skills training (CBSST) had significantly greater self-reported everyday functioning and coping skills

\footnotetext{
Abbreviations: $\mathrm{BIS}=$ Birchwood Insight Scale, CBSST $=$ cognitive-behavioral social skills training, $\mathrm{CBT}=$ cognitivebehavioral therapy, HAM-D = Hamilton Rating Scale for Depression, ILSS = Independent Living Skills Survey, SD = standard deviation, TAU = treatment as usual.

* Address correspondence to Lindsay C. Emmerson, PhD, c/o Eric Granholm, PhD; Department of Veterans Affairs San Diego Healthcare System (116B), 3350 La Jolla Village Drive, San Diego, CA 92161; 858-245-7200; fax: 858-6426416. Email: Icemmerson@msn.com

DOI:10.1682/JRRD.2009.04.0042
} 
knowledge than those who received treatment as usual (TAU) [9]. Improvements in everyday functioning and coping skills knowledge were maintained in the CBSST group compared with the TAU group at 12-month posttreatment follow-up [10].

The present study was a secondary analysis that sought to determine whether insight moderated the effect of CBSST on treatment outcomes in our clinical trial [9]. Given previous research demonstrating that greater insight is generally related to improved treatment outcomes in schizophrenia, we predicted that greater insight would moderate the effects of CBSST on functional outcomes. We suspected that the effectiveness of different treatment elements, from identifying dysfunctional performance beliefs to practicing social role playing, on functional outcomes would be affected by the level of awareness of having a mental illness that requires treatment. Specifically, individuals with greater awareness of illness may be more able to recognize their own maladaptive thoughts and social skills deficits that limit everyday functioning and may be more motivated to use skills taught in CBSST to improve their lives. Furthermore, since insight is a multidimensional construct, we wished to determine whether specific domains of insight (awareness of illness, relabeling of symptoms, or need for treatment) predicted benefit from CBSST. Building on prior research linking insight to hopelessness in schizophrenia $[1,6]$ and a growing literature on the impact of cognitivebehavioral therapy (CBT) for psychosis on hopelessness (for a review, see Wykes et al. [11]), we analyzed post hoc the role of hopelessness in the relationship between insight and functioning in CBSST to better understand the varying impact of greater insight on course of schizophrenia. Individuals who better understand their illness and lack hope in their ability to improve their lives may be less motivated to use the skills taught in CBSST to improve their everyday functioning.

\section{METHODS}

\section{Sample}

Community-dwelling middle-aged and older people (mean age 53 years; range 42-72 years) diagnosed with schizophrenia or schizoaffective disorder based on the Structured Clinical Interview for Diagnostic and Statistical Manual of Mental Disorders-Fourth Edition [12] were recruited between 1999 and 2003 from treatment and resi- dential settings through the University of California, San Diego, Advanced Center for Interventions and Services Research for Psychosis in Older Adults. Principal findings from this sample have been previously reported, including raw data for the outcome measures [9-10]. A subset $(n=62)$ of the total sample $(N=76)$ with complete insight ratings at baseline and 12-month follow-up is included in this article. No significant differences were found between participants included in this subsample and those excluded with respect to demographic variables (age, age of onset of illness, duration of illness, education, race [white vs other], residence [board and care vs other], sex) or clinical ratings (level of insight, everyday functioning, depressive symptoms).

\section{Study Design}

The San Diego Human Subjects Committee of the University of California approved this study. We obtained written informed consent from all participants. The study was a clinical trial in which participants (76\% male; 78\% Caucasian) were randomized to either TAU $(n=31)$ or TAU plus CBSST (hereafter referred to as CBSST, $n=31$ ) and treated for 6 months. No significant baseline group differences were found on any demographic or outcome variables, and assessors were blinded to treatment group.

\section{Measures}

The Birchwood Insight Scale (BIS) is an eight-item self-report measure with three insight subscales: Awareness of Illness (i.e., awareness of having a mental illness), Relabel (i.e., attribution of one's symptoms as part of one's disorder), and Need for Treatment [13]. The first two subscales include two items each, while the third includes four items, with all items being statements (e.g., "I am mentally well," "My doctor is right in prescribing medication for me") with possible responses of "agree," "unsure," or "disagree" that are scored on a 3-point Likerttype scale ranging from 0 to 2 . Subscale items are summed, and the Need for Treatment subscale is divided by 2, which accounts for having twice as many items, yielding a total score for each subscale. Total scores may be summed for a BIS total score (range $=0-12$ ), with higher scores indicating greater insight. The BIS is widely used in research on psychosis and has very good reliability, validity, and sensitivity to change [13].

The Independent Living Skills Survey (ILSS) is a self-report measure of basic functional living skills performed in the past month and was administered in an 
interview format [14]. A composite score was computed as the average of scores on five domains: appearance and clothing, personal hygiene, health maintenance, transportation, and leisure and community. In both younger [1415] and older patients [16] with schizophrenia, the ILSS has been shown to be a reliable, stable, and sensitive measure of functional deficit. We used the Hamilton Rating Scale for Depression (HAM-D) to assess depressive symptoms and hopelessness (item 27) [17].

\section{Interventions}

In TAU, participants continued in whatever care they were receiving and no medication guidelines were provided. The primary treatment target of CBSST was functioning [9]. Participants received 24 2-hour weekly group psychotherapy sessions with a half-hour lunch break (lunch was provided). Two repetitions of each of three modules ("thought-challenging," "asking for support," and "solving problems") were completed. A workbook that contained homework forms was provided. CBT components were developed specifically for people with schizophrenia (e.g., aids to compensate for cognitive impairment) [18-19]. A thorough description of CBSST is provided elsewhere [9-10].

Doctoral-level psychologists or senior graduate students in clinical psychology with at least master's level training and 2 years of clinical experience delivered the CBSST intervention. Groups were led by two therapists. Training and weekly supervision were provided, including review of session videotapes. We rated therapist competence using the Cognitive Therapy Scale for Psychosis [20] to rate 30 videotaped sessions that were randomly selected but stratified according to the module. An interclass correlation of 0.85 demonstrated good interrater reliability. The mean rating was $43 \pm 7$ standard deviation [SD]; a score of $\geq 39$ has previously been viewed as adequate technical competence in CBT for psychosis [21]. (Further values are expressed as mean $\pm \mathrm{SD}$, unless otherwise stated.)

\section{RESULTS}

At baseline, mean BIS scores were $9.0 \pm 1.7(n=31)$ and $8.7 \pm 2.7(n=31)$ for the CBSST and TAU groups, respectively; at the end of treatment, $8.4 \pm 2.4(n=28)$ and $9.4 \pm 2.6(n=31)$; and at 12-month follow-up, $8.8 \pm$ $2.1(n=31)$ and $8.8 \pm 2.3(n=31)$, respectively. BIS scores did not differ significantly between the groups at any time point (baseline: $t(60)=0.48, p>0.05$; end of treatment: $t(57)=1.45, p>0.05$; 12-month follow-up: $t(60)<0.01, p>0.05)$. We used multilevel modeling to examine treatment effects on the BIS. Model assumptions were checked, but no violations were found. The time $(t(60)=0.04, p>0.05)$, group $(t(60)=0.42, p>$ $0.05)$, and group by time interaction $(t(60)=0.05, p>$ 0.05 ) effects were not significant, so growth rates for CBSST and TAU were similar.

Linear regression models tested moderator relationships between baseline insight (BIS) and change in insight (difference between 12-month follow-up and baseline BIS scores) as predictors of ILSS at the 12-month follow-up assessment. Predictor variables in the models were group (coded CBSST $=0.5$; TAU $=-0.5$ ), baseline BIS, BIS change score, group by baseline BIS and group by BIS change score interactions, and baseline ILSS score as a covariate. The groups differed significantly at the 12-month follow-up ( $b=0.06, t(52)=2.30, p<0.05)$. A significant group by baseline BIS interaction $(b=$ $0.03, t(52)=2.12, p<0.05$ ) was also found, indicating that baseline insight moderated ILSS. No other predictors were significant.

To illustrate the moderator effect, the Figure shows the mean scores of the ILSS for the 12-month follow-up for high and low insight subgroups of each treatment group. "High" insight was defined as having a baseline BIS total median score of $>9$, and "low" insight defined as a score of $\leq 9$. This median split is consistent with the recommendation by Birchwood et al. that BIS total scores of 9 through 12 represent high insight [13]. CBSST participants with high insight had better functioning relative to TAU participants, but this finding was because participants in TAU with high insight had poorer functioning than those with low insight. For participants with high insight, CBSST significantly improved functioning compared with TAU, $t(25)=3.15, p<0.01$ ). This pattern suggests that high insight was associated with lower functioning but that CBSST mitigated the effect of high insight.

The significant group by BIS baseline interaction just described was further explored through post hoc linear regression models of the BIS subscales; the BIS variables were replaced with BIS subscale variables (Awareness, Relabel, and Need for Treatment). Only the group by BIS Need for Treatment interaction was significant (ILSS: $b=$ $0.11, t(52)=2.38, p<0.05)$. 


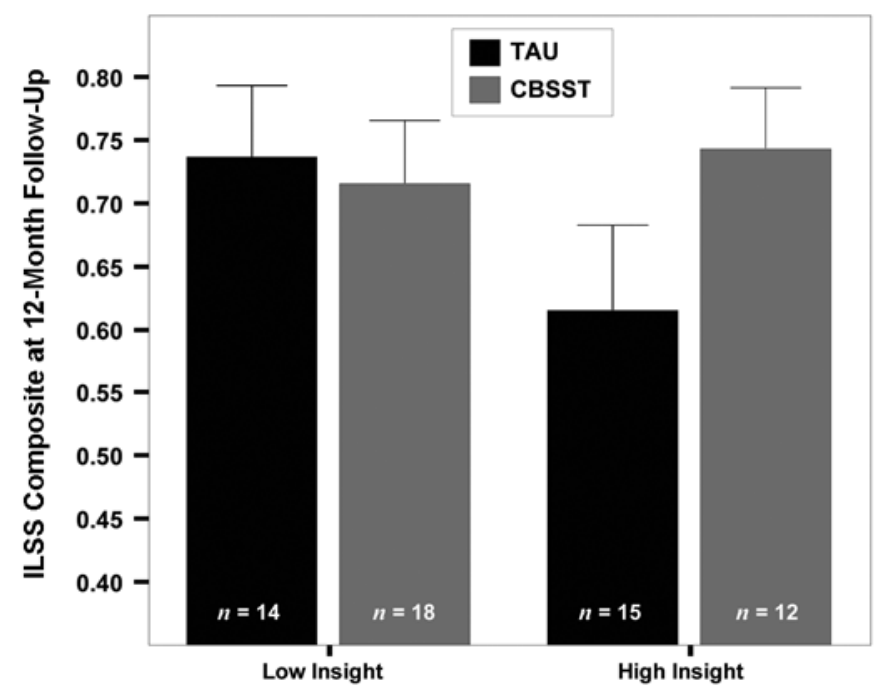

Figure.

Average Independent Living Skills Survey (ILSS) composite scores at 12-month follow-up for low insight ( $\leq 9$ on Birchwood Insight Scale) and high insight $(>9)$ middle-aged or older people with schizophrenia or schizoaffective disorder in each treatment group. Error bars are 95\% confidence interval. For high insight, participant scores in cognitive-behavioral social skills training (CBSST) were significantly $>$ those in treatment as usual (TAU), $p<0.01$.

Given the established relationship between high insight and depression in people with schizophrenia $[1,3]$, we conducted post hoc Chi-square and correlation analyses to explore the hypothesis that CBSST might have reduced the negative impact of insight-linked hopelessness on functioning compared with TAU, thus contributing to the moderation effect just described. In the total sample, greater insight (BIS) at follow-up was not significantly correlated with HAM-D total scores $(r=$ $0.19, p=0.15, n=62$ ), but was significantly correlated with greater hopelessness at follow-up (Spearman rank correlation $r=0.26, p=0.04, n=61){ }^{*}$ We used Chisquare analysis to evaluate the impact of treatment on the presence of any hopelessness in patients (1 on HAM-D item 27). The CBSST group showed a trend for having

\footnotetext{
* Corresponding analysis of the BIS subscales revealed the same pattern as seen for the total BIS for the Awareness subscale (nonsignificant correlation with HAM-D total: Spearman $r=0.22, p=0.08, n=$ 62; significant correlation with hopelessness: Spearman $r=0.28, p=$ 0.03, $n=61$ ), but the Relabel and Need for Treatment subscales were not related to HAM-D total or hopelessness $(p>0.05)$.
}

lower rates of hopelessness than the TAU group $\left(\chi^{2}=\right.$ 3.12, $p=0.08, n=61$ ), with double the rate of hopelessness in TAU relative to CBSST, and higher rates of hopelessness in patients with higher insight (CBSST: high $n=3$ of $12=25 \%$, low $n=3$ of $19=16 \%$; TAU: high $n=7$ of $14=50 \%$, low $n=5$ of $16=31 \%$ ).

\section{DISCUSSION}

With these secondary analyses of data from our randomized controlled trial of CBSST for middle-aged and older people with schizophrenia or schizoaffective disorder, we sought to determine whether insight moderated treatment outcome. We were particularly interested in the effect of insight on everyday functioning, which was the primary treatment target and outcome measure that improved significantly more with CBSST than TAU [910]. Consistent with our hypothesis, the present results suggest that insight moderated the effect of CBSST on functional outcome. People with high insight, especially those who believed they have an illness that requires treatment, had better everyday functioning in CBSST than in TAU. This finding suggests that people with higher insight might benefit the most in CBSST than in TAU, but no evidence was found to show that people with low insight should be excluded from CBSST (i.e., that CBSST is contraindicated for people with low insight). This group may benefit from CBSST as much as the high insight group on meaningful treatment targets other than everyday functioning. The everyday functioning of people in CBSST with high and low insight did not differ significantly.

Prior research has indicated that increased insight may be associated with more severe depression and hopelessness [1,3]. Older people who have suffered from schizophrenia for several decades may have faced many failure experiences and lifelong disability that could lead to hopelessness about attempting to improve functioning. In particular, participants who have accepted their mental illness diagnosis may internalize stigma and failure expectations prevalent in society today. Consistent with this speculation, post hoc exploratory analyses showed that across treatment groups, participants with higher insight were significantly more hopeless. A nonsignificant trend was also found showing lower rates of people with hopelessness at 12-month follow-up in CBSST than in TAU, with half as many people with hopelessness in 
CBSST than in TAU. These findings suggested that the typical negative impact of insight-linked hopelessness on functioning was reduced by elements of CBSST (e.g., identifying dysfunctional performance beliefs, practicing social role playing); however, these were post hoc analyses based on a single-item rating of hopelessness with a limited range of scores, so they should be replicated. If further research suggests that change in cognitive factors of depression (e.g., hopelessness, worthlessness, helplessness) mediate change in functional outcome, these factors may be important targets of CBT (i.e., the teaching of skills relevant to managing hopelessness, including increased activity level and challenging hopeless thoughts and dysfunctional performance beliefs).

The absence of a treatment effect on insight indicates that change in insight did not mediate outcome in CBSST [22]. Strengths of this study include treatment randomization, blind raters, well-matched groups at baseline on all variables, a manualized intervention, treatment fidelity monitoring, and good participant attendance and retention. CBSST did not specifically target insight but rather aimed to improve functioning. Also, this study was limited in that it involved older people with schizophrenia whose baseline levels of insight were high (BIS $8.86 \pm$ 2.23), thereby reducing room to improve insight. Other limitations of this study included small sample and subgroup sizes; exclusion of people with current substance dependence; lack of control for nonspecific therapist contact factors; and use of a brief self-report insight measure, with a limited range of scores for the subscales. More comprehensive interview scales might result in a different moderation effect, especially for multidimensional aspects of insight.

\section{CONCLUSIONS}

Improving functional behaviors represents a meaningful change in the quality of life of individuals with schizophrenia, as well as a socially relevant treatment goal, especially for older people who generally have more stable symptoms and fewer hospitalizations but still have poor functioning [23]. Insight, especially insight into need for treatment, moderated the relationship between treatment group and functioning, such that CBSST offset the negative effect of insight on functioning with TAU. Post hoc analyses showed that reduction of insight-linked hopelessness might have accounted for the positive effect of CBSST on functioning compared with TAU. These findings along with prior research on the relationship between insight and hopelessness and depression [1,3] suggest that hopelessness may be an important clinical target in CBT for schizophrenia.

\section{ACKNOWLEDGMENTS}

\section{Author Contributions:}

Study concept and design: L. C. Emmerson, E. Granholm, D. V. Jeste. Acquisition of data: E. Granholm, D. V. Jeste.

Analysis and interpretation of data: L. C. Emmerson, E. Granholm. Drafting of manuscript: L. C. Emmerson.

Critical revision of manuscript for important intellectual content:

E. Granholm, J. R. McQuaid, D. V. Jeste.

Statistical analysis: P. C. Link.

Financial Disclosures: The authors have declared that no competing interests exist.

Funding/Support: This material was based on work supported by the Department of Veterans Affairs, Veterans Health Administration, Rehabilitation Research and Development Service (Eric Granholm, PhD; Principle Investigator, Department of Psychiatry), and by National Institute of Mental Health grants R01 MH-61381 and 1P30 MH-006248.

Additional Contributions: Portions of this research were presented at the 2007 International Congress on Schizophrenia Research in Colorado Springs, Colorado.

Participant Follow-Up: The authors do not plan to inform participants of the publication of this study.

\section{REFERENCES}

1. Mintz AR, Dobson KS, Romney DM. Insight in schizophrenia: A meta-analysis. Schizophr Res. 2003;61(1):75-88. [PMID: 12648738] DOI:10.1016/S0920-9964(02)00316-X

2. Francis JL, Penn DL. The relationship between insight and social skill in persons with severe mental illness. J Nerv Ment Dis. 2001;189(12):822-29. [PMID: 11794575] DOI:10.1097/00005053-200112000-00003

3. Lysaker PH, Bell MD, Bryson GJ, Kaplan E. Insight and interpersonal function in schizophrenia. J Nerv Ment Dis. 1998;186(7):432-36. DOI:10.1097/00005053-199807000-00008

4. Fenton WS, Blyler CR, Heinssen RK. Determinants of medication compliance in schizophrenia: Empirical and clinical findings. Schizophr Bull. 1997;23(4):637-51. [PMID: 9366000]

5. Smith TE, Hull JW, Goodman M, Hedayat-Harris A, Willson DF, Israel LM, Munich RL. The relative influences of symptoms, insight, and neurocognition on social adjustment in 
schizophrenia and schizoaffective disorder. J Nerv Ment Dis. 1999;187(2):102-8. [PMID: 10067950] DOI:10.1097/00005053-199902000-00006

6. Lysaker PH, Roe D, Yanos PT. Toward understanding the insight paradox: Internalized stigma moderates the association between insight and social functioning, hope, and selfesteem among people with schizophrenia spectrum disorders. Schizophr Bull. 2007;33(1):192-99. [PMID: 16894025] DOI:10.1093/schbul/sbl016

7. Amador XF, Friedman JH, Kasapis C, Yale SA, Flaum M, Gorman JM. Suicidal behavior in schizophrenia and its relationship with awareness of illness. Am J Psychiatry. 1996; 153(9):1185-88. [PMID: 8780423$]$

8. Tarrier N, Haddock G, Lewis S, Drake R, Gregg L; SoCRATES Trial Group. Suicide behaviour over 18 months in recent onset schizophrenic patients: The effects of CBT. Schizophr Res. 2006;83(1):15-27. [PMID: 16460916] DOI:10.1016/j.schres.2005.12.846

9. Granholm E, McQuaid JR, McClure FS, Auslander LA, Perivoliotis D, Pedrelli P, Patterson T, Jeste DV. A randomized, controlled trial of cognitive behavioral social skills training for middle-aged and older outpatients with chronic schizophrenia. Am J Psychiatry. 2005;162(3):520-29.

[PMID: 15741469]

DOI:10.1176/appi.ajp.162.3.520

10. Granholm E, McQuaid JR, McClure FS, Link PC, Perivoliotis D, Gottlieb JD, Patterson TL, Jeste DV. Randomized controlled trial of cognitive behavioral social skills training for older people with schizophrenia: 12-month follow-up. J Clin Psychiatry. 2007;68(5):730-37. [PMID: 17503982]

11. Wykes T, Steel C, Everitt B, Tarrier N. Cognitive behavioral therapy for schizophrenia: Effect sizes, clinical models, and methodological rigor. Schizophr Bull. 2008;34(3): 523-37. [PMID: 17962231]

DOI:10.1093/schbul/sbm114

12. First MB, Spitzer RL, Gibbon M. Structured clinical interview for DSM-IV Axis I disorders: Patient edition, SCID-P. New York (NY): New York State Psychiatric Institute; 1998.

13. Birchwood M, Smith V, Drury V, Healy J, Macmillan F, Slade M. A self-report Insight Scale for psychosis: Reliability, validity and sensitivity to change. Acta Psychiatr Scand. 1994;89(1):62-67. [PMID: 7908156]

DOI:10.1111/j.1600-0447.1994.tb01487.x

14. Wallace CJ, Liberman RP, Tauber R, Wallace J. The Independent Living Skills Survey: A comprehensive measure of the community functioning of severely and persistently mentally ill individuals. Schizophr Bull. 2000;26(3):631-58. [PMID: 10993403]

15. Vaccaro JV, Pitts DB, Wallace CJ. Functional assessment. In: Liberman RP, editor. Handbook of psychiatric rehabilitation. New York (NY): Macmillan; 1992. p. 78-94.

16. Perivoliotis D, Granholm E, Patterson TL. Psychosocial functioning on the Independent Living Skills Survey in older outpatients with schizophrenia. Schizophr Res. 2004; 69(2-3):307-16. [PMID: 15469202]

DOI:10.1016/j.schres.2003.09.012

17. Hamilton M. Development of a rating scale for primary depressive illness. Br J Soc Clin Psychol. 1967;6(4):278-96. [PMID: 6080235]

18. Beck AT, Rector NA. Cognitive approaches to schizophrenia: Theory and therapy. Annu Rev Clin Psychol. 2005;1: 577-606. [PMID: 17716100]

DOI:10.1146/annurev.clinpsy.1.102803.144205

19. Kingdon DG, Turkington D. Cognitive therapy of schizophrenia. New York (NY): Guilford Press; 2008.

20. Haddock G, Devane S, Bradshaw T, McGovern J, Tarrier N, Kinderman P, Baguley I, Lancashire S, Harris N. An investigation into the psychometric properties of the Cognitive Therapy Scale for Psychosis (CTS-Psy). Behav Cogn Psychother. 2001;29(2):221-33.

DOI:10.1017/S1352465801002089

21. Sensky T, Turkington D, Kingdon D, Scott JL, Scott J, Siddle R, O’Carroll M, Barnes TR. A randomized controlled trial of cognitive-behavioral therapy for persistent symptoms in schizophrenia resistant to medication. Arch Gen Psychiatry. 2000;57(2);165-72. [PMID: 10665619] DOI:10.1001/archpsyc.57.2.165

22. Kraemer HC, Wilson GT, Fairburn CG, Agras WS. Mediators and moderators of treatment effects in randomized clinical trails. Arch Gen Psychiatry. 2002;59(10):877-83. [PMID: 12365874] DOI:10.1001/archpsyc.59.10.877

23. Jeste DV, Alexopoulos GS, Bartels SJ, Cummings JL, Gallo JJ, Gottlieb GL, Halpain MC, Palmer BW, Patterson TL, Reynolds CF 3rd, Lebowitz BD. Consensus statement on the upcoming crisis in geriatric mental health: Research agenda for the next 2 decades. Arch Gen Psychiatry. 1999; 56(9):848-53. [PMID: 12884891]

DOI:10.1001/archpsyc.56.9.848

Submitted for publication April 8, 2009. Accepted in revised form June 23, 2009. 